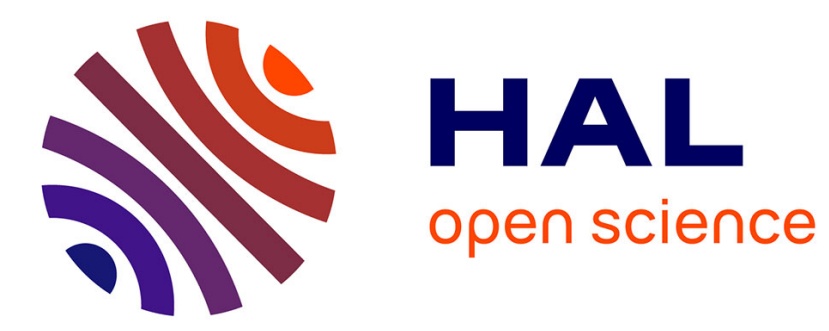

\title{
Physical interpretation of the rheological behaviour of amorphous polymers through the glass transition
}

\author{
J. Perez, J.Y. Cavaille, S. Etienne, C. Jourdan
}

\section{To cite this version:}

J. Perez, J.Y. Cavaille, S. Etienne, C. Jourdan. Physical interpretation of the rheological behaviour of amorphous polymers through the glass transition. Revue de Physique Appliquée, 1988, 23 (2), pp.125-135. 10.1051/rphysap:01988002302012500 . jpa-00245755

HAL Id: jpa-00245755

https://hal.science/jpa-00245755

Submitted on 1 Jan 1988

HAL is a multi-disciplinary open access archive for the deposit and dissemination of scientific research documents, whether they are published or not. The documents may come from teaching and research institutions in France or abroad, or from public or private research centers.
L'archive ouverte pluridisciplinaire $\mathbf{H A L}$, est destinée au dépôt et à la diffusion de documents scientifiques de niveau recherche, publiés ou non, émanant des établissements d'enseignement et de recherche français ou étrangers, des laboratoires publics ou privés. 


\title{
Physical interpretation of the rheological behaviour of amorphous polymers through the glass transition
}

\author{
J. Perez, J. Y. Cavaille, S. Etienne and C. Jourdan \\ Groupes d'Etudes de Métallurgie Physique et de Physique des Matériaux, U. A. CNRS 341, I.N.S.A., \\ 69621 Villeurbanne Cedex, France
}

(Reçu le 8 octobre 1986, révisé le 14 août 1987 et le 23 novembre 1987, accepté le 24 novembre 1987)

\begin{abstract}
Résumé. - Ce travail a pour objectif de décrire le comportement mécanique des polymères amorphes soumis à une sollicitation cyclique au voisinage de la transition vitreuse (relaxation $\alpha$ ). A cette fin, une théorie de la déformation non élastique est établie à partir d'un modèle de mobilité moléculaire. La structure du polymère amorphe est assimilée à un empilement compact de monomères dans lequel se trouvent des sites de désordre caractérisés par un excès d'enthalpie et d'entropie. Ces sites, appelés « défauts », sont dénombrés par un calcul thermodynamique usuel en métallurgie physique. On voit ensuite comment l'application d'une contrainte donne naissance, sur les sites de moindre résistance, à des microdomaines cisaillés (Mdc) qui sont à l'origine de la déformation non élastique. Celle-ci comporte deux termes : l'un anélastique (réversible) dû à la formation des Smd et associé au temps $\tau_{1}$, l'autre viscoplastique (non réversible) dû à la propagation des Smd et associé au temps $\tau_{2}$. Les temps $\tau_{1}$ et $\tau_{2}$ sont reliés à un temps unique $\tau_{\max }$, par l'application du concept de hiérarchisation des mouvements corrélés récemment introduit par Palmer et al. On obtient finalement les modules dynamiques $G^{\prime}, G^{\prime \prime}$ et $\tan \varphi$. Tous les paramètres sont accessibles à la mesure et ont un sens physique précis : densité de défauts $C_{\mathrm{d}}$, temps caractéristique $\tau_{\mathrm{mr}}$ associé à $\tau_{\max } \sim \tau_{\mathrm{mol}}$ qui est un temps de diffusion monomérique et deux coefficients $h$ et $k$ exprimant l'intensité des effets de corrélation existant d'une part lors des mouvements moléculaires initiaux (expansion réversible des $\mathrm{Mdc}$ ) et d'autre part lors des mouvements à plus longue distance (recombinaison des lignes bordant les Mdc). Des comparaisons sont faites avec des résultats expérimentaux, montrant la détermination des différents paramètres physiques. Ainsi, cette théorie rend compte aussi bien des valeurs élevées (et variables avec T) de l'énergie d'activation apparente du pic $\alpha$, que des lois exponentielles étendues (Kohlrausch ou Williams-Watt) décrivant le même pic.
\end{abstract}

\begin{abstract}
The purpose of the present work is to predict, from physical bases, the mechanical behaviour of amorphous polymers resulting from the application of a periodic stress through the glass transition range $(\alpha$ relaxation). A theory of the non elastic deformation is developed from a model of molecular mobility. The amorphous polymer is described in terms of a close packing of monomers in which there are sites corresponding to density fluctuations : these sites, exhibit an excess of enthalpy (and entropy) and are hereafter called « defects ». The concentration of defects is calculated from thermodynamic arguments usual in physical metallurgy. The application of a stress results in the nucleation of shear-microdomains (Smd) around the defects where the resistance to shear is weaker. Thus, the non elastic deformation has two components : one is anelastic due to the nucleation of the Smd (characteristic time $\tau_{1}$ ), the other is viscoplastic due to the expansion of the Smd until annihilation of the line bordering them (time $\tau_{2}$ ). Both $\tau_{1}$ and $\tau_{2}$ are related to a unique characteristic time $\tau_{\max }$ thanks to the application of the hierarchical (or series) correlation concept introduced earlier by Palmer et al. The dynamic modulus $\left(G^{\prime}\right.$ and $\left.G^{\prime \prime}\right)$ and $\tan \varphi$ are calculated. The parameters appearing in the equations thus obtained can be known from independent experimental ways and all have a precise physical meaning: the concentration of defects $C_{\mathrm{d}}$, the mechanical relaxation time $\tau_{\mathrm{mr}}$ linked to $\tau_{\max } \sim \tau_{\text {mol }}$ which is a life time for monomer diffusion and two coefficients, $k$ and $h$, indicating the effectiveness of correlation effects existing, on the one hand, during preliminary molecular movements (reversible expansion of Smd) and, on the other hand, during longer distance movements implying the annihilation of lines bordering the Smd. After determining the different physical parameters the comparison between theoretical and experimental results is made. Thus the physical origin of stretched exponentials (Kohlrausch or Williams-Watt's equation) as well as the high values of the apparent activation energy (which, moreover depend on $T$ ) can be explained.
\end{abstract}




\section{Introduction.}

There have been numerous investigations on the viscoelastic properties of amorphous polymers [1-3] and two temperature ranges can be distinguished.

i) At $T>T_{\mathrm{g}}$ (rubbery state), the behaviour of the material is explained in terms of entropic elasticity and chain reptation [4]. Beyond a critical length, chains are entangled. After an observation time $t_{\text {exp }}>\tau_{\mathrm{e}}$, the properties of the system depend on the fluctuations of the entanglement lattice: hence $\tau_{\mathrm{e}}$ is the relaxation time due to the loss of entanglement of chains $\left(\tau_{\mathrm{e}}=\eta_{0} / G_{\mathrm{c}}\right.$ with $\eta_{0}$ : zero stress viscosity and $G_{\mathrm{c}}$ : rubbery modulus.

ii) At $T<T_{\mathrm{g}}$ (glassy state), the cohesion of amorphous polymer is mainly due to intermolecular interaction: each monomer is linked not only to monomers of the same chain but to neighbouring monomers constituting a matrix surrounding it. Hence, the main obstacles to conformation changes are energy barriers reflecting the interaction between each monomer and the surrounding matrix.

Between those both ranges, the glass transition occurs and the corresponding viscoelastic properties are generally described with the equations of rheology. Nevertheless such an approach is phenomenological and implies a great number of parameters in terms of relaxation spectrum $H(\tau)$ (or retardation spectrum $L(\tau)$ ) which is not easily related to physical concepts. Although these properties depend obviously on the molecular mobility, they are not easily related to microstructural parameters. In a previous work [5] an attempt was made in order to interpret on physical bases the behaviour of amorphous polymers in the glass temperature range. In such a model, the concept of « defect » was introduced. The rheological properties were considered as resulting from the nucleation of shearmicrodomains (Smd) in these defects (time $\tau_{1}$ ) followed by the stress induced diffusional expansion of Smd until the lines bordering two neighbouring Smd are annihilated (time $\tau_{2}$ ). Using the concept of hierarchically correlated molecular movement, it is now possible to relate both phenomena to a unique characteristic time having a physical meaning and easy to obtain experimentally [6].

Thus, the purpose of this paper is to introduce this concept of correlated molecular movement into the theory quoted above [5] and to obtain a complete physical description of the rheological properties of amorphous polymers.

\section{Physical basis (summary).}

In reference [5], the physical bases of the interpretation of the rheological behaviour of amorphous polymers around $T_{\mathrm{g}}$ were given and only a summary will be presented here.
2.1 MICROSTRUCTURAL ASPECT AND MOLECULAR MOBILITY (assumption 1). - The polymer can be considered as a close packing of monomers whose rigidity is largely dominated by the intermolecular forces but some disordered sites may exist. At $T>T_{\mathrm{g}}$, these sites are continuously redistributed due to thermal fluctuations but at $T<T_{\mathrm{g}}$, they are frozen. Such sites, in concentration $C_{\mathrm{d}}$, will be hereafter called « defects ».

In order to calculate $C_{\mathrm{d}}$, it is assumed that there are two types of monomers :

i) Those (in number $N_{\mathrm{A}}-N_{\mathrm{d}}$ ) forming a close packing with their neighbours thus presenting the lowest level of inter and intramolecular bonds $\left(N_{\mathrm{A}}\right.$ : Avogadro's number).

ii) Those (in number $N_{\mathrm{d}}$ ) forming with their first neighbours a more disordered packing, thus having an increment of free enthalpy :

$$
\Delta G_{\mathrm{F}}=\Delta H_{\mathrm{F}}-T \Delta S_{\mathrm{F}}
$$

with $\Delta H_{\mathrm{F}}$ : enthalpy increment due to broken Van der Waals bonds and excited intramolecular bonds ; $\Delta S_{\mathrm{F}}$ is the entropy increment related to the different possible states of intramolecular bonds i.e. the entropy of formation of a defect.

The number $N_{\mathrm{d}}$ (i.e. $\left.C_{\mathrm{d}}=N_{\mathrm{d}} / N_{\mathrm{A}}\right)$ is obtained by minimizing the function :

$$
\Delta G=N_{\mathrm{d}} \Delta G_{\mathrm{F}}-T \Delta S_{\mathrm{M}}
$$

with $\Delta S_{\mathrm{M}}=k_{\mathrm{B}} \log \left(N_{\mathrm{A}} ! /\left(\left(N_{\mathrm{A}}-N_{\mathrm{d}}\right) ! N_{\mathrm{d}} !\right)\right)$ : entropy of mixing $N_{\mathrm{d}}$ defects among $N_{\mathrm{A}}$ positions.

At $T>T_{\mathrm{g}}$, the material is in a metastable equilibrium so that a Boltzmann distribution can be considered and we obtain :

$$
C_{\mathrm{d}}=\frac{1}{1+\exp \left(-\Delta S_{\mathrm{F}} / k_{\mathrm{B}}\right) \exp \left(\Delta H_{\mathrm{F}} / k_{\mathrm{B}} T\right)} .
$$

Such an approach is useful as it is possible to relate $\Delta H_{\mathrm{F}}$ and $\Delta S_{\mathrm{F}}$ to the value of $T_{\mathrm{g}}$ and $\Delta C_{\mathrm{p}}=$ $C_{\mathrm{p}}$ (liquid) $-C_{\mathrm{p}}$ (glass). For instance, it was found [5] $\Delta H_{\mathrm{F}}=0.114 \mathrm{eV} ; \Delta S_{\mathrm{F}}=2.32 k_{\mathrm{B}}$ and $\Delta H_{\mathrm{F}}=$ $0.060 \mathrm{eV} ; \Delta S_{\mathrm{F}}=2.30 k_{\mathrm{B}}$ in the cases of amorphous selenium and polybutadiene respectively. The variation of $C_{\mathrm{d}}$ with temperature has also been given (Ref. [5], Fig. 3).

Molecular movements obviously occur in these defects. Recalling Adam and Gibbs' theory [7], the probability for a transition from a configuration to another, is given by :

$$
P \sim \exp \left(-Z^{*} \Delta \mu / k_{\mathrm{B}} T\right)
$$

with $Z^{*}=S^{*} N_{\mathrm{A}} / \Delta S(T)$ : number of monomers constituting the region where a cooperative movement occurs ( $S^{*}$ minimum configuration entropy necessary for such a transition; $\Delta \mu$ : height of the 
energy barrier related to this transition; $\Delta S(T)$ : molar excess entropy of the disordered matter).

By assuming that the excess entropy is included in defects, it is possible to identify $\Delta S(T)$ to $\Delta S_{\mathrm{F}} \cdot N_{\mathrm{A}} \cdot C_{\mathrm{d}}$ and one has :

$$
P \sim \exp \left(-S^{*} \Delta \mu / \Delta S_{\mathrm{F}} C_{\mathrm{d}} K_{\mathrm{B}} T\right) .
$$

Taking $\Delta \mu^{*}=\Delta \mu S^{*} / \Delta S_{\mathrm{F}}$, we can calculate the lifetime for an elementary monomer movement :

$$
\tau_{\mathrm{mol}}=\tau_{0} \exp \left(\Delta \mu * / C_{\mathrm{d}} k_{\mathrm{B}} T\right)
$$

and the monomeric diffusion coefficient :

$$
D=\nu_{0} \lambda^{2} \exp \left(-\Delta \mu * / C_{\mathrm{d}} k_{\mathrm{B}} T\right)
$$

with $\nu_{0}=1 / \tau_{0}=k_{\mathrm{B}} T / h_{\mathrm{p}} ; \lambda$ : mean distance of displacement of a monomer during an elementary process of diffusion.

It happens that these equations are similar to those of Adam and Gibbs [7], $C_{\mathrm{d}}$ being the order parameter instead of $\Delta S(T)$, both being linearly connected.

Thus, at $T>T_{\mathrm{g}}$, there are entropy fluctuations and monomers are alternatively either close-packed or forming disordered regions with their neighbours, i.e. defects. At $T<T_{\mathrm{g}}$, those defects are frozen leading to local zones of high molecular mobility or « islands of mobility » [8].

\subsection{NON ELASTIC DEFORMATION OF AMORPHOUS} POLYMERS AT HIGH TEMPERATURE $\quad\left(T \geqslant 0.7 T_{\mathrm{g}}\right)$ (assumption 2). - We have proposed previously [9-11] that the basic deformation mechanism in amorphous solids, is the nucleation of shear microdomains (Smd). The nucleation occurs under the effect of the applied stress and is thermally assisted. A general case of Smd has been illustrated as follows : as shear initiates along a planar surface $S$, a cooperative atomic rearrangement occurs on both sides, inside a volume bounded by the curved surface $\Sigma$. The intersection between $\Sigma$ and $S$ defines the curve $C_{\mathrm{n}}$ which separates the part $S_{1}$ of $S$ where shear has occured from the non sheared part of $S$. In mechanics of continuous media, the line $C_{\mathrm{n}}$ is a dislocation loop ; in amorphous solids, dislocations, as far as this concept is valid, would be of the Somigliana type [11]. Some nucleation rate has been calculated by several authors but the results appear to be unrealistic as such a nucleation can occur only in regions where resistance to shear is appreciably weaker than in the rest of the material. Such soft sites may be regarded as the «defects» evoked above. The thermomechanical activation of a defect (mean time $\tau_{1}$ ) may lead to the formation of a Smd. When the stress is removed, the solid recovers its previous configuration, and this corresponds to the anelastic behaviour. In order to obtain plastic deformation, the growth of the Smd must be necessarily invoked.
But the line $C_{\mathrm{n}}$ being a Somigliana dislocation i.e. a sessile defect, such a growth needs a diffusional mechanism. This growth covers a distance (mean time $\tau_{2}$ ) at which the line $C_{\mathrm{n}}$ loses its identity by combination with other similar lines formed from the neighbouring defects, and becomes ineffective. This leads to a viscoplastic behaviour. Following those hypothesis, the number of defects $n(t)$ giving a Smd at time $t$, obeys the following equation [11] :

$$
\frac{\mathrm{d} n(t)}{\mathrm{d} t}=-\frac{n(t)-n(\infty)}{\tau_{1}}+\frac{n(0)-n(t)}{\tau_{2}} .
$$

Where $n(t)=n(0)$ at $t=0$ when the stress is applied and $n(\infty)$ is the equilibrium population of activated defects corresponding to the stress $\sigma$. Hence, the first term of the right hand side gives the nucleation rate of Smd and the second term corresponds to the rate of annihilation of lines bordering Smd until the defect, being again in its previous state, is able to be activated once more thus forming a new local shear.

After integration and introduction of the parameters $\Delta \gamma$ and $v_{\mathrm{a}}[11]$, the equation (2) gives the compliance (non elastic component) :

$$
\begin{aligned}
J(t)=A\left\{\frac{\tau_{2}\left(\tau_{2}-\tau_{1}\right)}{\left(\tau_{1}+\tau_{2}\right)^{2}} \times\right. \\
\left.\quad \times\left[1-\exp \left(-\frac{t}{\tau}\right)\right]+\frac{2 t}{\tau_{1}+\tau_{2}}\right\}
\end{aligned}
$$

with $1 / \tau=1 / \tau_{1}+1 / \tau_{2} ; A=\alpha \Delta \gamma f v_{\mathrm{a}} v_{1} N_{0} / k_{\mathrm{B}} T$ $(\Delta \gamma$ shear strain in a Smd; $f$ Schmid factor; $v_{\mathrm{a}}$ and $v_{1}$ respectively activation volume and volume of matter concerned by the Smd; $N_{0}$ : number of defects per unit volume i.e. $N_{0} v_{1} \simeq C_{\mathrm{d}} ; \alpha$ : constant about 0.1 [11]).

2.3 DYNAMIC COMPLIANCE. - In reference [5], a possible distribution of parameters $\tau_{1}$ and $\tau_{2}$ was taken into account resulting in an expression for $J(t)$ so complicated that its Fourier transform was approximated with the empirical equation :

$$
J^{*}(i \omega)=\frac{1}{G_{\mathrm{I}}}\left\{1+\left(i \omega \tau_{\mathrm{M}}\right)-h+\frac{C}{1+(i \omega \tau)^{k}}\right\}
$$

with $G_{\mathrm{I}}$ : unrelaxed modulus ;

$$
\begin{aligned}
\tau_{\mathrm{M}} & =\left(\tau_{1}+\tau_{2}\right) / 2 A G_{\mathrm{I}} \\
C & =\tau_{2}\left(\tau_{2}-\tau_{1}\right) A G_{\mathrm{I}} /\left(\tau_{1}+\tau_{2}\right)^{2}
\end{aligned}
$$

the parameters $h$ and $k$ (between 0 and 1) were considered to characterize the distribution width of $\tau_{M}$ (i.e. mainly $\tau_{2}$ ) and $\tau\left(\right.$ i.e. mainly $\tau_{1}$ ) respectively.

The relation (4) was used to compare experimental data and calculated curves $G^{\prime}(\omega)$ and $G^{\prime \prime}(\omega)$ (storage and loss modulus respectively) in the case of polystyrene, selenium and polybutadiene. In order 
to obtain a good agreement between both, it was necessary to take $\tau_{2}$ not too different of $\tau_{1}$ although the latter is more widely distributed than the former. Furthermore, the temperature dependence of both times had to be similar. Such features are not implied in the physical hypothesis mentioned before ; so, a new pattern must be involved to complete the preceding description and to obtain a new expression for describing the dynamic behaviour of amorphous polymers; in the following, such an expression, although similar to equation (4), will be deduced from the physical bases included in this new pattern.

\section{Correlated molecular movement in condensed matter (assumption 3).}

In reference [11], equation (3) was used to « explain » experimental data by using a suitable choice of the weight distribution of $\tau_{1}$ and $\tau_{2}$. However, this approach is microscopically arbitrary and does not explain the universality of Kohlrausch factor $\exp \left[-(t / \tau)^{b}\right]$ with $b$ : parameter between 0 and 1 . So, instead of the picture of parallel relaxation, in which each degree of freedom $i$ relaxes independently with characteristic time $\tau_{i}$, Palmer et al. [12] considered a series interpretation, involving a distribution having a microscopic source in the correlations between different degrees of freedom. In other words, a hierarchy of degrees of freedom, from fast to slow is involved: the fastest might correspond to single-atom motion; other atoms, or groups of atoms, might only be able to move appreciably when several of the fastest happen to be placed in just the right way, leaving « free volume » or weakening a bond.

Following Palmer et al. [12], let us consider a discrete series of levels $n=1,2, \ldots$ with the degrees of freedom in level $n$ represented by $N_{n}$ Ising spins. Each spin in level $n$ is only free to change its state if a condition on some spins in level $n-1$ is satisfied, such as $\mu_{n-1}$ spins in level $n-1$ attain a particular state of their $2^{\mu_{n-1}}$ possible ones. The average relaxation time $\tau_{n}$ will be related by :

$$
\tau_{n}=\tau_{n-1} * 2^{\mu_{n-1}}
$$

giving :

$$
\tau_{n}=\tau_{1} \exp \left(\sum_{1}^{n-1} \mu_{\ell} \operatorname{Ln} 2\right)
$$

It was considered by Palmer et al. that $\mu_{\ell}$ decreases when the level of the system goes up and one of the possibilities discussed by the authors was:

$$
\mu_{\ell} \operatorname{Ln} 2=\mu_{1 \ell^{-p}}
$$

(the value of $p$ being given further).
Alternatively, we suggest a constant time $t_{0}$ is required for the system to go from the level $\ell$ to the level $\ell+1$. Hence it takes a time $t_{\ell}=\ell t_{0}$ for the system to go from the level 0 to the level $\ell$, and $\ell$ may be replaced in equation (6a) by $t_{\ell} / t_{0}$ :

$$
\mu_{\ell} \operatorname{Ln} 2=\mu_{1}\left(t_{\ell} / t_{0}\right)^{-p}
$$

In other words, introducing the duration of level change makes the connection with the microstructure of the material to appear, since changing the level of the system implies time dependent atomic movements.

Thus, by using equation (6b) instead of (6a), equation (5) is transformed into :

$$
\tau(t)=\tau_{1} \exp \left[\mu_{1} \sum\left(t_{\ell} / t_{0}\right)^{-p}\right] .
$$

Equation (7) is similar to equation (5) and postulate (c) of reference [12] : $\tau(t)$ (as $\tau_{n}$ of Eq. (5)) is the characteristic time corresponding to the excitation of the system in such a way that in the $n$th first level $\left(n=t / t_{0}\right)$, changes i.e. atomic movements occur ; this excitation is obtained after surmounting sequentially a succession of free energy barriers separating increasing energy levels.

By replacing the sum by an integral, equation (7) can be transformed into :

$$
\begin{aligned}
\tau(t) & =\tau_{1} \exp \left[\mu_{1} \int_{1}^{n}\left(t / t_{0}\right)^{-p} d\left(t / t_{0}\right)\right] \\
& =\tau_{1} \exp \left[\mu_{1}\left(1-n^{1-p}\right) /(p-1)\right](\text { with } p \neq 1)
\end{aligned}
$$

$0<\mu_{1}<1$ may be regarded as a structural parameter characterizing the correlation between the different atomic movements in the glass which, as a solid, is a strongly interacting system. For $\mu_{1}=0$ the matter surrounding the first moving atom is not sensitive to such a movement and $\tau(t)$ reduces to $\tau_{1}$. For $\mu_{1}=1$, the primary movement induces numerous correlated atomic movements and $\tau(t)$ can reach the maximum value $\tau_{\max }$. We furthermore expect such features to be relevant to glassy materials when $\tau_{1}<\tau_{\max }<\infty$; in reference [12], $\tau_{\max }$ was the limit of $\tau_{n}$ when $n \rightarrow \infty$ with $p=1+\varepsilon$ ( $\varepsilon$ is positive and much smaller than 1). In those conditions, the present description may be compared to the work of Ngai and White [13] who proposed an unified theory of the low frequency dynamic response of condensed matter : the relaxation of a primary species (side group, chain segment and, more generally, a dipole...) is followed by an interaction with correlated states presenting low energy excitations. To underline this comparison, let us calculate $\tau(t)$ from equation (8) by developing it in series around $\varepsilon=0$; at the first order (which corresponds to $p=1$ ), we have : 


$$
\tau(t)=\tau_{1}\left(t / t_{0}\right)^{\mu_{1}}
$$

which is equivalent to the result of Ngai and White (Eq. (9) of Ref. [13]) :

$$
\tau(t)=\tau_{1}^{\prime}\left(t / t_{\mathrm{c}}\right)^{n}
$$

with $\tau_{1}^{\prime}=\tau_{1} \exp n \gamma ; \gamma=0.577$ (Euler constant) ; $n<1 ; t_{\mathrm{c}}=h / E_{\mathrm{c}}$ and $E_{\mathrm{c}}$ is the upper « cutoff » of the correlated state excitation.

By taking into account the condition $\tau(t)=$ $\tau_{\max }$ when $t=\tau_{\max }$, equation (9) leads to :

$$
\tau_{\max }=\left(\tau_{1} / t_{0}^{\mu_{1}}\right)^{1 /\left(1-\mu_{1}\right)}
$$

implying :

$$
\tau(t)=\tau_{\max } 1-\mu_{1 t} \mu_{1} .
$$

Thus, this result could correspond to figure $1 \mathrm{a}$ : the preliminary atomic movement needs the time $\tau_{1}$ but this movement results in a reorganisation of the neighbouring matter corresponding to the whole duration $\tau_{\max }$.

Let us apply this assumption about correlated movement to the picture recalled in part 2.2; figure $1 \mathrm{~b}$ then shows what could be the microstructural content of the correlated movement hypothesis. The preliminary molecular movement (duration $\tau_{1}$ ) induces hierarchically correlated monomer movements resulting first in a local shear (Smd) ; moreover, as long as stress is applied, the loop expands around this Smd thanks to those correlated movements and the duration of the whole process is $\tau_{\max }$. If the matter behaves as a simple packing of nearly spherical units, $\tau_{\max }$ would be equal to $\tau_{2}$ which is the time needed for the loop to lose its identity (this is roughly the situation with metallic and oxide glasses); but in the case of polymers, the elemental units (monomers) form chains which interact one each other (entanglements, graft nodes, interfaces). The consequence is that loop annihilation is followed by some local movements near these obstacles, thus implying a second

(a)

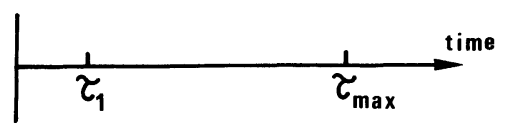

(b)

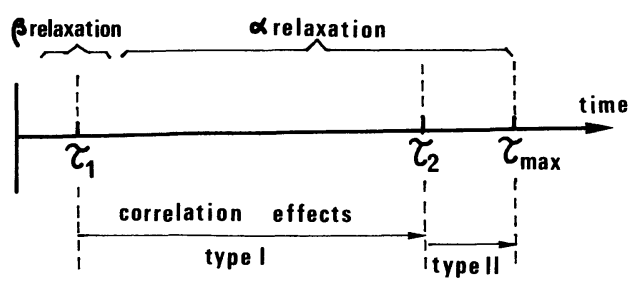

Fig. 1. - Time scale for 1a) preliminary atomic movements $\left.\left(\tau_{1}\right), 1 \mathrm{~b}\right)$ correlated atomic movements (application of stress at $t=0$ ). type of correlation effect. Then, as shown in figure $1 \mathrm{~b}$, correlation effects of type I and II may be distinguished; the former is concerned by the topological necessity to rearrange nearly spherical units (monomers) in order to obtain a propagation of shear; the latter corresponds to correlation effects at longer distance implying a topological constraint at the level of obstacles such as entanglements, nodes or interfaces.

In these conditions, instead of using $\tau_{1}$ and $\tau_{2}$ as introduced in part 2.2, one must take into account equation (11) leading to :

$$
\begin{gathered}
\tau_{1}(t)=\tau_{\max } 1-\mu_{1 t} \mu_{1} \\
\tau_{2}(t)=\tau_{\max } 1-\mu_{2 t} \mu_{2}
\end{gathered}
$$

with $\mu_{1}$ and $\mu_{2}$ : factors corresponding to correlation effects of type I and II respectively. From both preceding definitions and figure $1 \mathrm{~b}$, it appears that $\mu_{1}>\mu_{2}$; moreover, $\mu_{2}=0$ would correspond to $\tau_{2}(t)=\tau_{\max }$ i.e. the occurrence of only the correlation effect of type $\mathrm{I}$.

It is worthy to notice that, if experimental time $t_{\text {exp }}$ is shorter than $\tau_{\max }$, glassy systems clearly break ergodicity so that equilibrium distribution in configurationnal space are not usable. On the contrary, if $t_{\exp }>\tau_{\max }$ the system is ergodic, so that statistical equilibrium laws become valid and relaxation phenomena require pure exponential relations.

To sum up, several behaviours can be expected (see Fig. 1b) :

i) $t_{\text {exp }} \gg \tau_{\max }$ : shear is irreversible (viscoplasticity) and no memory of the deformation is left but the rubbery effect.

ii) $\tau_{\max }>t_{\exp }>\tau_{2}$ : shear is irreversible but owing to the necessary compatibility of deformation, some internal stresses appear.

iii) $\tau_{2}>t_{\exp }>\tau_{1}$ : shear is reversible : the shear strain is recovered when the stress is suppressed (anelasticity).

\section{Non elastic deformation and dynamic modulus.}

Due to correlation effects, $\tau_{1}$ and $\tau_{2}$ in equation (2) must be replaced by $\tau_{1}(t)$ and $\tau_{2}(t)$ given by equations (12a) and (12b) respectively. Hence, equation (2) is not any more easy to integrate. A simplification can be made as $\tau_{2}(t)>\tau_{1}(t)$ so that equation (2) is equivalent to a set of two independent equations; then, as developed in appendix, we have :

i) The anelastic behaviour (nucleation and reversible expansion of Smd)

$$
J_{\text {anel }}(t)=A\left\{1-\exp \left[-\frac{1}{1-\mu_{1}}\left(\frac{t}{\tau_{\max }}\right)^{1-\mu_{1}}\right]\right\}
$$

which is obtained as a short time approximation. 
ii) The viscoplastic behaviour (loop annihilation) described by :

$$
J_{\text {viscopl. }}(t)=\frac{A}{1-\mu_{2}}\left(\frac{t}{\tau_{\max }}\right)^{1-\mu_{2}}
$$

corresponding to a long time approximation.

iii) The global compliance :

$$
\begin{array}{r}
J(t)=\frac{1}{G_{\mathrm{I}}}+A\left\{1-\exp \left[-\frac{1}{1-\mu_{1}}\left(\frac{t}{\tau_{\max }}\right)^{1-\mu_{1}}\right]\right\}+ \\
+\frac{A}{1-\mu_{2}}\left(\frac{t}{\tau_{\max }}\right)^{1-\mu_{2}} .
\end{array}
$$

Such a relation is to be compared to equation (3) despite the fact that coupling effects between anelastic (short time $t \ll \tau_{\max }$ ) and viscoplastic (long time $t \leq \tau_{\max }$ behaviours have been neglected. In agreement with this approximation, the anelastic term of equation (14) can be developed in series. So, the compliance $J(t)$ is a sum of terms with which it is easy to calculate the Fourier transform leading to :

$$
\begin{array}{r}
J^{*}(i \omega)=\frac{1}{G_{\mathrm{I}}}\left\{1+\frac{\Gamma(k+1)}{k} A G_{\mathrm{I}}\left(i \omega \tau_{\max }\right)^{-k}+\right. \\
\left.+\frac{\Gamma(h+1)}{h} A G_{\mathrm{I}}\left(i \omega \tau_{\max }\right)^{-h}\right\}
\end{array}
$$

which can be written :

$J^{*}(i \omega)=\frac{1}{G_{\mathrm{I}}}\left\{1+H\left(i \omega \tau_{\mathrm{mr}}\right)^{-h}+\left(i \omega \tau_{\mathrm{mr}}\right)^{-k}\right\}$

with : $k=1-\mu_{1} ; h=1-\mu_{2} ; \Gamma(k+1) \simeq 1$ and $\Gamma(h+1) \simeq 1$ : gamma functions ; $\tau_{\mathrm{mr}}=$ $\left(A G_{\mathrm{I}} / k\right)^{-1 / k} \tau_{\max }$ : mechanical relaxation time; $H=k^{h / k} h^{-1}\left(A G_{\mathrm{I}}\right)^{1-h / k}$.

Equation (15) is very similar to equation (4) but there is only one characteristic time : the mechanical relaxation time $\tau_{\mathrm{mr}}$.

Furthermore, equation (15) is formally to be compared with the rheological expression corresponding to the modified limited biparabolic model as discussed by Decroix [14] but in equation (15) all the parameters have a physical meaning: $\tau_{\mathrm{mr}}$ is proportional to the molecular mobility; $k$ and $h$ $(0<k<h<1)$ give the intensity of correlated effects of type I and II respectively and $H$ depends on $h, k$ and $A$ which is proportional to the defect density.

To obtain a complete description of the dynamic modulus, it is necessary to take into account, i) the rubbery elasticity and, ii) the loss of entanglement and the reptation of chains. Figure 2 explains how to introduce both phenomena : the rubbery modulus is $G_{\mathrm{c}}=\rho R T / M_{\mathrm{e}}$, with $\rho$ : specific mass $; M_{\mathrm{e}}:$ mean value of the mass of the sub-chain between two neighbouring entanglements; $R=N_{\mathrm{A}} k_{\mathrm{B}}$. This modulus corresponds to the spring $\mathrm{CD}$ so that equation (15) can be transformed into $\left(G_{I}\right.$ of Eq. (15) becoming now $G_{\mathrm{I}}-G_{\mathrm{c}}$ ):

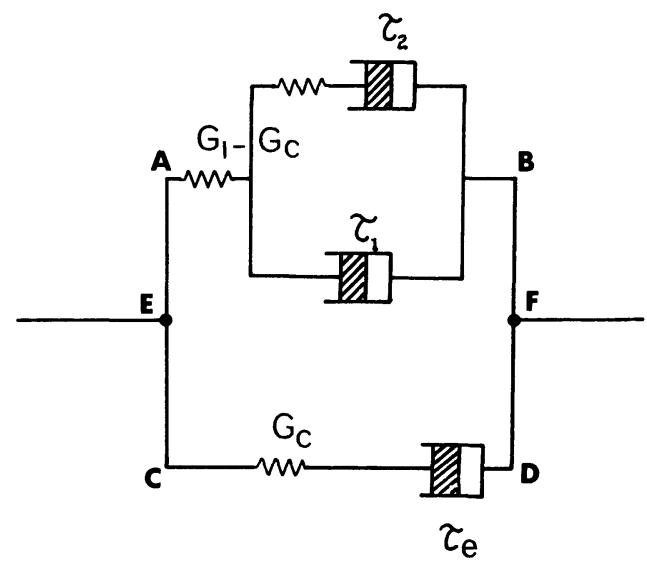

Fig. 2. - Rheological diagram describing the dynamic response and correspondance with physical parameters :

- $G_{I}$ : modulus at infinite frequency $\left(G_{I}>G_{\mathrm{c}}\right)$ (unrelaxed)

- $\tau_{1}$ : characteristic time for Smd nucleation

- $\tau_{2}$ : characteristic time for annihilation of lines bordering Smd

- $G_{\mathrm{c}}$ : rubbery modulus

- $\tau_{\mathrm{e}}$ : characteristic time for the loss of entanglement of chains.

$$
\begin{aligned}
G_{\mathrm{EF}}^{*}(i \omega)= & G_{\mathrm{C}}+\left(G_{\mathrm{I}}-G_{\mathrm{C}}\right) / \\
& /\left[1+H\left(i \omega \tau_{\mathrm{mr}}\right)^{-h}+\left(i \omega \tau_{\mathrm{mr}}\right)^{-k}\right] .
\end{aligned}
$$

Furthermore, the modulus $G_{\mathrm{C}}$ can be relaxed through the unentanglement of chains. As shown by Doi and Edwards [15], the rubbery modulus is then given by :

$$
G_{\mathrm{CD}}=G_{\mathrm{C}} \sum_{q} 8 / \pi^{2} q^{2} \exp \left(-t / \tau_{\mathrm{e}}\right)
$$

$\tau_{\mathrm{e}}$ is the tube disengagement time given by :

$$
\tau_{\mathrm{e}}=L^{2} \xi_{\mathrm{m}} /\left(\pi^{2} q^{2} k_{\mathrm{B}} T\right)
$$

$L$ length of the tube along which each chain reptates; $\xi_{\mathrm{m}}$ molecular friction coefficient ; $q$ : odd number. Thus, in the case of a dynamic experiment, equation (16) can be written, with $G_{\mathrm{I}} \gg G_{\mathrm{C}}$

$$
\begin{aligned}
G_{\mathrm{EF}}^{*}(i \omega)= & G_{\mathrm{c}} \sum_{j} \frac{i \omega \tau_{\mathrm{e} j}}{1+j \omega \tau_{\mathrm{e} j}} g_{j}+ \\
& +\frac{G_{\mathrm{I}}}{1+H\left(i \omega \tau_{\mathrm{mr}}\right)^{-h}+\left(i \omega \tau_{\mathrm{mr}}\right)^{-k}}
\end{aligned}
$$

The sum $\sum_{j}$ results from the different values of $q$; moreover, one may think on the one hand that $L$ has not a single value and, on the other hand, the characteristics of strands are also distributed [1]. In such conditions, one can only choose an arbitrary $\operatorname{sum} \sum_{j}$ (square, or triangular or Gaussian) with $\sum g_{j}=1\left(g_{i}\right.$ : statistic weight of each process $\left.\tau_{\mathrm{e} j}\right)$. 
Finally equation (18) gives $G^{\prime}, G^{\prime \prime}$ and $\tan \varphi=$ $G^{\prime \prime} / G^{\prime}$ but actually, in the following, equation (16) may be used as far as measurements are done near the glass transition temperature for which $\tau_{\mathrm{mr}} \ll$ $\tau_{\mathrm{e}}$.

\section{Application of the model ; discussion.}

In this part, the model presented here above will be applied to describe experimental data obtained either in isothermal condition (frequency scanning) or as a function of temperature (temperature scanning) then a discussion will be developed in order to underline all the possibilities included in the model.

5.1 ISOTHERMAL EXPERIMENTS. - The variation of $G^{\prime}, G^{\prime \prime}$ and $\tan \varphi$ as a function of $\omega$ can be calculated from equation (16) and compared to experimental master curves. At a first sight, the number of parameters is important ; actually, each of them can be obtained independently of each others :

- firstly, experimental master curves as usually drawn [1c] give directly $G_{\mathrm{I}}$ (limit value of $G^{\prime}$ for $\omega \rightarrow \infty$ ), $G_{\mathrm{C}}$ (inflection point of the curve $G^{\prime}(\omega)$, corresponding to the rubbery state), $\tau_{\mathrm{e}}$ (end of the rubbery plateau).

- Then, the COLE-COLE diagram $G^{\prime \prime}=$ $f\left(G^{\prime}\right)$, gives $h, k$ and $A$; this is shown schematically in figure 3.

- Lastly, $\tau_{\mathrm{mr}}$ is chosen to have a good coincidence between calculated and experimental peaks of $\tan \varphi=f(\omega)$.

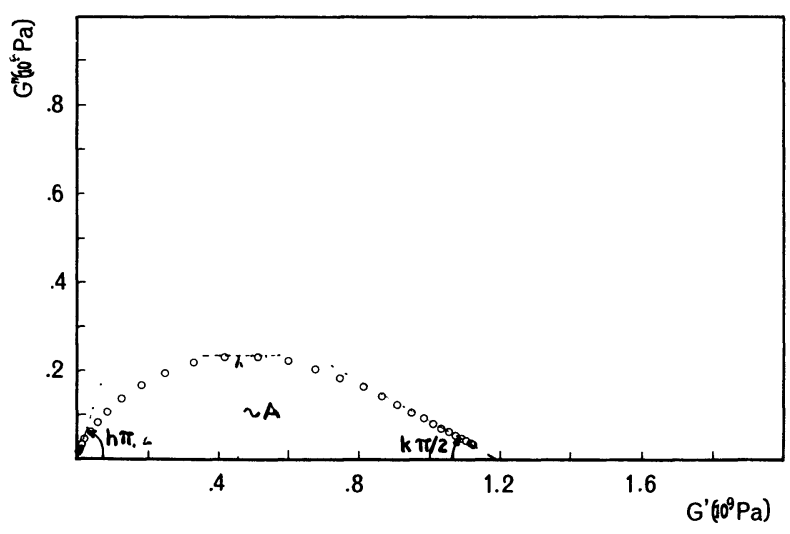

Fig. 3. - Schematic Cole-Cole diagram. The slopes of tangents at $\omega \rightarrow \infty$ and $\omega \rightarrow 0$ give $k$ and $h$ respectively ; the height of Cole-Cole curve leads to $A$ (Eq. (15)-(18)).

This comparison is made with master curves given in reference [16] for a polybutadiene (Fig. 4) and in reference [17] for a polyvinylacetate (Fig. 5) and for a polybutylacrylate (Fig. 6).

Such an agreement between theory and experimental data was mentionned before [5] but only one

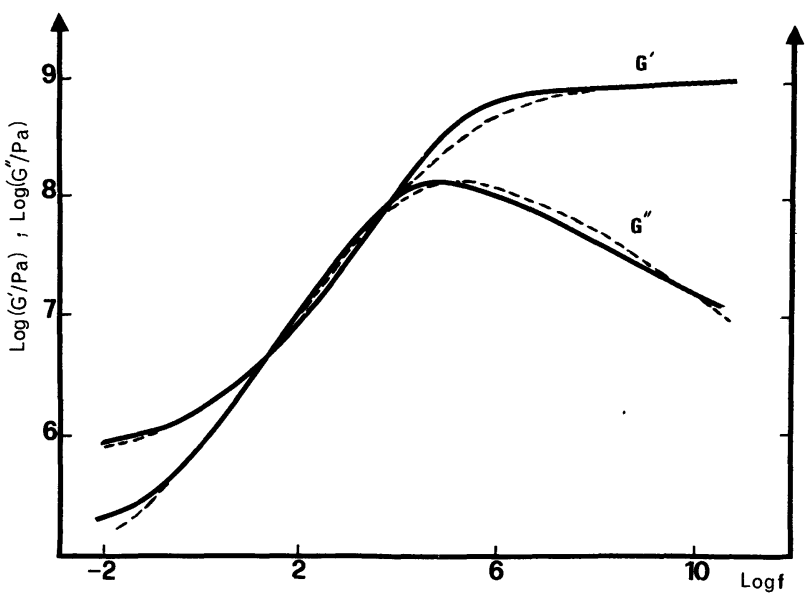

Fig. 4. - Solid lines : experimental master curves obtained with a polybutadiene $\left(T_{\mathrm{g}} \simeq 153 \mathrm{~K}\right)$ [16]. Dotted lines : curves calculated using equations (16), (18) with $G_{1}=10^{9} \mathrm{~Pa}, \quad G_{\mathrm{c}}=8.2 \times 10^{5} \mathrm{~Pa}, \quad H=4.8, \quad h=0.54$, $k=0.24$. (Measurements have been done between 153 and $203 \mathrm{~K}$ in the frequency range $10^{-2}-250 \mathrm{~Hz}$; the maximum scattering in $\log (G / \mathrm{Pa})$ is \pm 0.1 .)

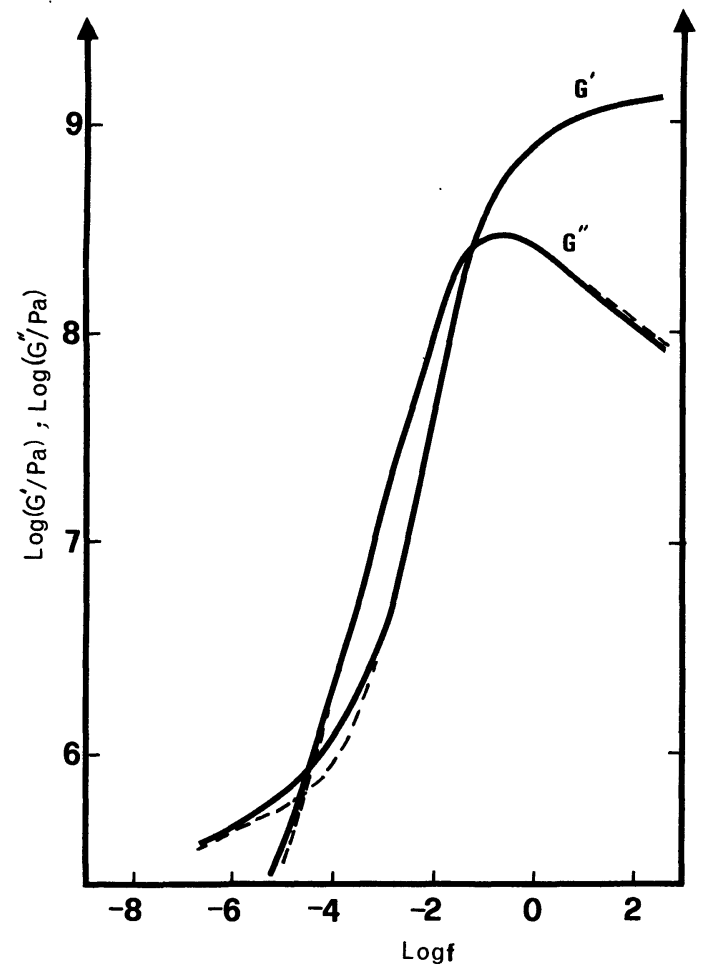

Fig. 5. - Solid lines : experimental master curves obtained with a polyvinylacetate $\left(T_{\mathrm{g}} \simeq 301 \mathrm{~K}\right)$ [17]. Dotted lines : curves calculated using equations (16), (18) with $G_{\mathrm{I}}=2 \times 10^{9} \mathrm{~Pa}, G_{\mathrm{c}}=3.3 \times 10^{5} \mathrm{~Pa}, H=0.25, h=0.91$, $k=0.27$. (Measurements have been done between 305 and $322 \mathrm{~K}$ in the frequency range $5 \times 10^{-5}-5 \mathrm{~Hz}$; the maximum scattering in $\log (G / \mathrm{Pa})$ is \pm 0.05 .

characteristic time is needed in the present work; furthermore, the problem of distribution is solved without further hypothesis ; thus, i) $k$ governs 


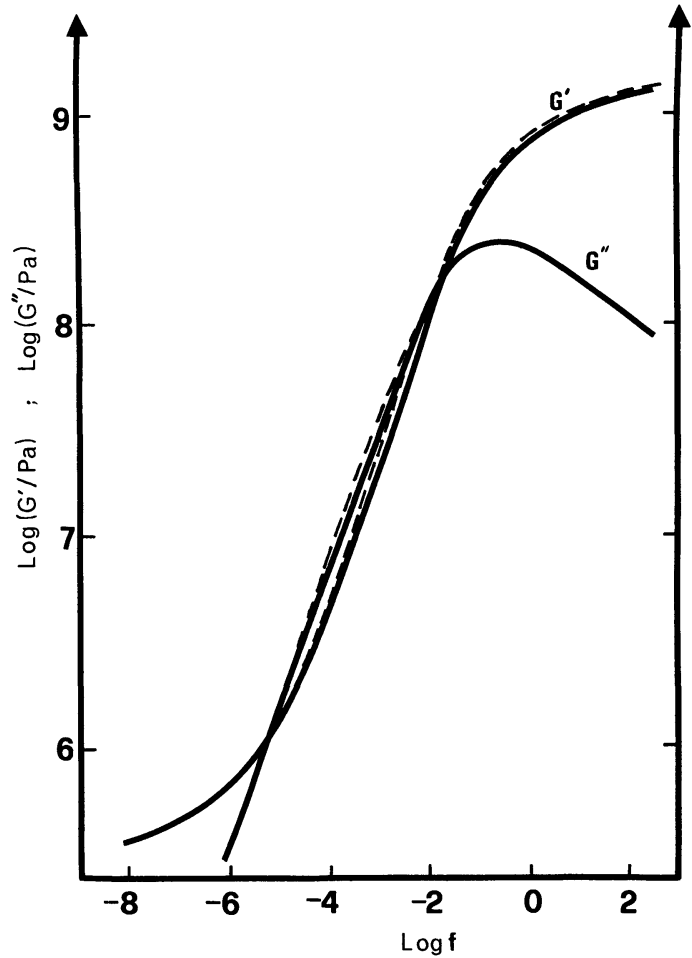

Fig. 6. - Solid lınes : experimental master curves obtained with polybutylacrylate $\left(T_{\mathrm{g}} \simeq 250 \mathrm{~K}\right)$ [17]. Dotted lines : curves calculated using equations (16), (18) with $G_{\mathrm{I}}=2 \times 10^{9} \mathrm{~Pa}, \quad G_{\mathrm{c}}=8 \times 10^{5} \mathrm{~Pa}, \quad H=0.4, \quad h=0.70$, $k=0.27$. (Measurements have been done between 217 and $245 \mathrm{~K}$ in the frequency range $5 \times 10^{-5}-5 \mathrm{~Hz}$; the maximum scattering in $\log (G / \mathrm{Pa})$ is \pm 0.05 .

mainly the high frequency part of the master curves and, ii) $h$ governs the height and the width of the peak $\tan \varphi=f(\omega)[18]:(\tan \varphi)_{\max }$ is higher when $h$ approaches unity (e.g. for polystyrene, $h=0.95$ and $\left(\tan \varphi_{\max }\right.$ is about 6$)$ and decreases with $h$ (e.g. for, epoxy resin or semi-crystalline polypropylene, $h$ is lower than 0.7 leading to $(\tan \varphi)_{\max }$ lower than 1$)$. This is in agreement with the assumed physical origin of $h:$ in the former case (polystyrene), correlation effects of type II (see part 3 ) are limited to entanglements ; in the latter case these correlation effects imply either graft nodes (epoxy resin) or interfaces (semi-crystalline polypropylene).

5.2 VARIATION OF DYNAMIC MODULUS WITH TEMPERATURE. - In order to interpret spectra obtained through temperature scanning with equations (16)(18), the temperature variation of $\tau_{\mathrm{mr}}$ must be precised. In this purpose, two cases can be distinguished :

i) At $T<T_{\mathrm{g}}$, the polymer can be considered as a solid and, in the case of experiments made in isoconfigurational conditions, the approach based on correlation effect leads to equations (10) and (12a) with $\mu_{1}$ being constant.
In equation (10), comparable to that proposed by Ngai and White [13] and referred to second universality, $\tau_{1}$ is a characteristic time of the preliminary molecular movement leading to the formation of the Smd; let us consider that this molecular process is connected to the intramolecular rotational freedom degree of the smallest sub-chain atoms further along the chain. Such a possibility, well known as «crankshaft » process [19], might be responsible for the $\beta$ relaxation. Hence, in equation (10), $\tau_{1}$ might be similar to the $\beta$ relaxation time with :

$$
\tau_{1}=\tau_{0} \exp \left(U_{1} / k_{\mathrm{B}} T\right) .
$$

It is worthy to notice that $\tau_{1}$ is probably distributed due to the disordered state of the matter; nevertheless such a distribution is not so wide than that induced by correlated effects as schematically shown in figure $1 \mathrm{~b}$. In other words, the relaxation time spectrum could be formed of two regions (Fig. 1b) : one slightly distributed around $\tau_{1}$, mean value corresponding to $\beta$ relaxation, and the other, stretched between $\tau_{1}$ and $\tau_{\max }$.

ii) At $T>T_{\mathrm{g}}$, the matter is in a metastable equilibrium (supercooled liquid) : the structure (i.e. the coefficient of correlation) is temperature dependent but it is not so obvious to have a relation between $\mu_{1}$ (or $\mu_{2}$ ) and $T$. Then the best way to describe temperature dependence of rheological properties of polymeric glasses might be to relate the characteristic time $\tau_{\mathrm{mr}}$ to the life time for the movement $\tau_{\text {mol }}$, as the temperature dependence of the latter can be known via the temperature dependence of the defect density (Eq. (1a) and (1b)).

On the other hand, $\tau_{\max }$ can be identified to the diffusion time of the species moving in order to relax internal stress fields due to the evolution of Smd. In a similar way that in reference [11], a good approximation can be given by :

$$
\tau_{\max }=l_{\mathrm{c}} / v
$$

with $l_{\mathrm{c}}$ : mean diffusion length; $v$ is the mean velocity of diffusing species and can be found from Einstein relation :

$$
v=D F / k_{\mathrm{B}} T
$$

$F$ is the force acting on the moving species :

$$
F=E_{\mathrm{el}} / l_{\mathrm{c}}
$$

with $E_{\mathrm{el}}$ : elastic energy of stress field line singularity per molecular length.

From equations $(1 b, c)$ and $(20 a, b, c)$, one obtains :

$$
\tau_{\max }=\left(l_{\mathrm{c}} / \lambda\right)^{2}\left(k_{\mathrm{B}} T / E_{\mathrm{el}}\right) \tau_{\operatorname{mol}}
$$

which means that $\tau_{\max }$ is of the order of magnitude of $\tau_{\text {mol }}$. 
To sum up, i) recalling that the characteristic time accessible to experiments is :

$$
\tau_{\mathrm{mr}}=\left(A G_{\mathrm{I}} / k\right)^{-1 / k} \tau_{\max }=\delta \tau_{\max } \quad(\text { see Eq. (15)) }
$$

and, ii) taking into account equations (10) and (19), we have :

$$
\begin{aligned}
& \text { - at } T>T_{\mathrm{g}} \text { : } \\
& \tau_{\mathrm{mr}}=\tau_{0}^{\prime} \exp \left(\Delta \mu * / C_{\mathrm{d}} k_{\mathrm{B}} T\right) \text { with } \tau_{0}^{\prime}=\delta \tau_{0} \\
& \text { - at } T<T_{\mathrm{g}} \text { : } \\
& \tau_{\mathrm{mr}}=\tau_{0}^{\prime \prime} t_{0}^{-(1-k) / k} \exp \left(U_{1} / k k_{\mathrm{B}} T\right) \\
& \text { with } \quad \tau_{0}^{\prime \prime}=\delta \tau_{0}^{1 / k} \text {. }
\end{aligned}
$$

There is only one adjustable parameter which is $\Delta \mu *\left(T>T_{\mathrm{g}}\right)$ or $t_{0}\left(T<T_{\mathrm{g}}\right)$.

Let us apply those equations to experimental results obtained with a polybutadiene : in the reference [16] the dynamic modulus was obtained at frequencies between $10^{-3}$ and $10^{3} \mathrm{~Hz}$ in a temperature range higher than $T_{\mathrm{g}}$ so that we need in this case only equation (22a) ; by taking $\Delta \mu *=0.06 \mathrm{eV}$ and $C_{\mathrm{d}}$ calculated as indicated in part 2.1 , the variation of $\tau_{\mathrm{mr}}$ with temperature is shown in the upper part of figure 7. The variation of $G^{\prime}$ and $\tan \varphi$ with temperature, calculated with equation (16) with the corresponding value of $\tau_{\mathrm{mr}}$, is reported in the lower part of the same figure. Those curves are compared to experimental results of Salvia obtained at different temperatures in isothermal conditions [16] :

- $(\operatorname{Tan} \varphi)_{\max }=1.1 \quad$ (experimental value : between 1.15 and 1.20).

- The non symetric form of the calculated $\tan \varphi$ peak is the same as that of the experimental curve.

- $G^{\prime}$ decreases of 3 orders of magnitude when the temperature is increased of $46 \mathrm{~K}$ (experimentally, the same $G^{\prime}$ decrease need $50 \mathrm{~K}$ ).

- The apparent activation energy defined by

$$
E_{\mathrm{a}}=-k_{\mathrm{B}} d\left(\ln \tau_{\mathrm{mr}}\right) d(1 / T)
$$

is found to vary between 1 and $2.5 \mathrm{eV}$ in agreement with experimental values as shown in figure 7 (diagram inserted in the upper part).

It is worthy to notice that equation (22a) explains the temperature dependence of $E_{\mathrm{a}}$ which appears to be the consequence of the temperature dependence of $C_{\mathrm{d}}$ : thus a possible molecular origin of FulcherTamman-Vogel's equation (and WLF law) could be naturally established instead of introducing arbitrarily such an equation as in reference [12].

At temperatures lower than $T_{\mathrm{g}}$, although $C_{\mathrm{d}}$ is constant (isoconfigurational conditions), high values of $E_{\mathrm{a}}$ are generally mentionned : for instance, in the case of polystyrene, $E_{\mathrm{a}}$ was experimentally found about $3 \mathrm{eV}$ [20] ; equations (19) and (22b) lead to

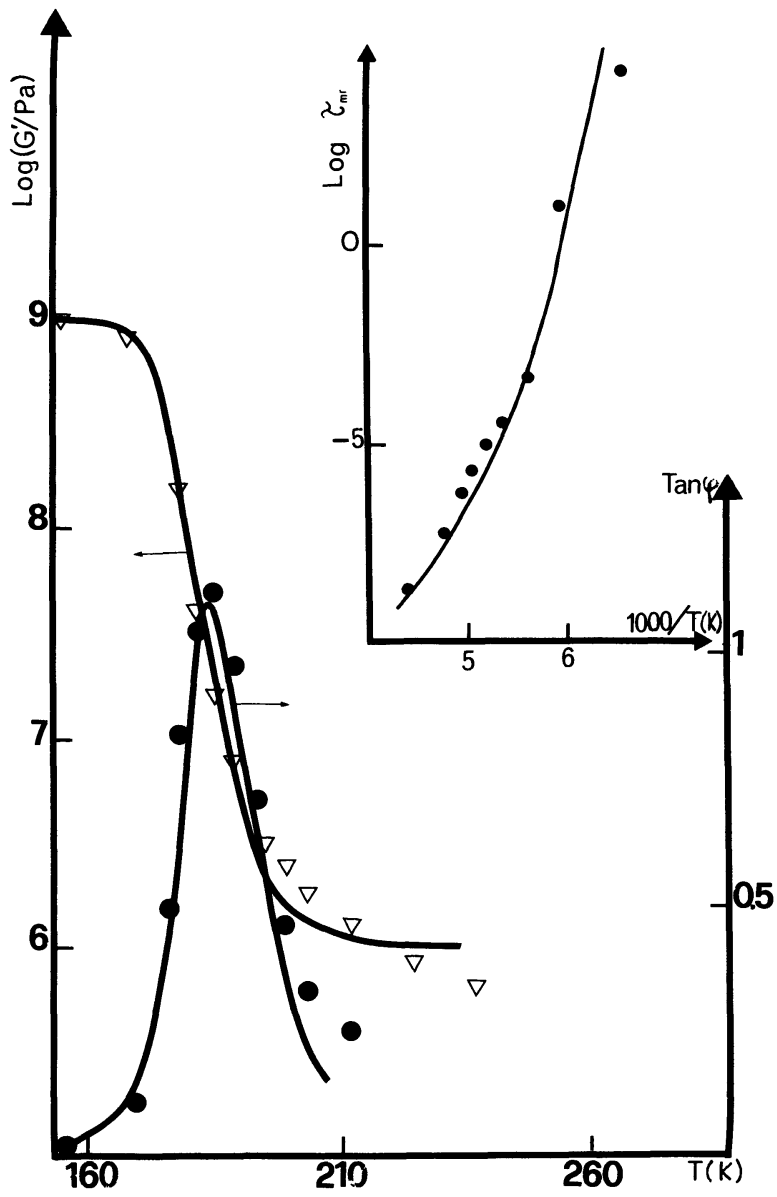

Fig. 7. - Curves $G^{\prime}$ and $\tan \varphi=G^{\prime \prime} / G^{\prime}$ versus temperature calculated using i) equation (16) and ii) the variation of $\tau_{\mathrm{mr}}$ with temperature shown in the upper part (see text for parameters). Experimental points correspond to polybutadiene [16]. Solid lines : calculated.

$E_{\mathrm{a}}=U_{1} / k$ i.e. $3.2 \mathrm{eV}$. Similarly, the values of preexponential factor lower than $10^{-30} \mathrm{~s}$ (given even in isoconfigurational conditions) are explained by equation (22b).

To conclude this point, let us remark that high values of apparent activation energy was previously interpreted in terms of a model of cooperative site changes [22] ; but, beyond the fact that equations of this model are neither very convenient nor predictive, the stretched exponential decay and Fulcher's equation are not discussed.

5.3 CREEP AND STRESS RELAXATION TESTS. - The detailed calculation of creep and stress relaxation curves is beyond the scope of this paper. Nevertheless it is worthy to notice the following points :

i) Equation (14) shows that the short time non elastic compliance varies as $\left(t / \tau_{\max }\right)^{1-\mu_{1}}$. Considering that $1-\mu_{1}$ is not too different from 0.3 in the case of polystyrene [20], it appears that the form of the creep in this region is Andrade creep, in 
agreement with results of Plazek [21]. For longer time, the rubbery effect must be taken into account but the experimental slope $\mathrm{d} \log J(t) / \mathrm{d} \log t$ is about 0.9 , which can be compared to the value of $h$ $[18,20]$.

ii) By inverse Fourier transformation of equation (16), the stress relaxation function may be obtained as :

$$
G(t)=G_{\mathrm{c}}+\frac{G_{\mathrm{I}}-G_{\mathrm{c}}}{1+H\left(t / \tau_{\mathrm{mr}}\right)^{h}+\left(t / \tau_{\mathrm{mr}}\right)^{k}} .
$$

This equation has nearly the same form as empirical formulations as mentionned in reference [1(b)].

\section{Conclusion.}

The theory presented here is mainly based on the hypothesis of thermomechanical nucleation of Smd from among defects. Thus the low temperature (high frequency) side of the $\alpha$ relaxation peak could correspond to localized molecular movements (anelastic behaviour and structural parameters $k, A$, $G_{\mathrm{I}}$ and $\left.\tau(t)\right)$; on the other hand, the high temperature (low frequency) side of this peak could correspond to longer distance movements (viscoplastic behaviour and parameters $\left.h, A, G_{\mathrm{c}}\right)$.

Both characteristic times $\tau(t)$ and $\tau_{\mathrm{rm}}$ have been related to the time life $\tau_{\text {mol }}$ of a monomer movement thanks to the hierarchic correlation assumption which, moreover, leads to the Kolhrausch factor: from this point of view, the present work is linked to papers recently published $[23,24]$ in order to give a physical bases to stretched exponentials generally invoked in fitting experimental relaxation curves obtained with glassy solids. The ideas proposed here may be viewed as an improvement of concepts presented in Palmer's paper, i) using assumptions clearly formulated in terms of microstructure and, ii) leading to equations easily usable to be compared with experimental data.

To conclude, in such a picture the so-called « glass transition " is conditionned (through $\tau_{\mathrm{mr}}$ ) by both intramolecular (through $\Delta \mu$ ) and intermolecular (through $C_{\mathrm{d}}$ ) forces. In this way, the model here proposed differs noticeably from other models in the same field, which are generally phenomenological in their nature.

The consequences of such an analysis about the nature of glass transition, the structural relaxation in glasses, the connection between $\beta$ and $\alpha$ relaxations... might be important. This will be developed elsewhere.

\section{Appendix.}

Let us consider $N_{0}$ defects per unit volume, statistically distributed between 2 populations : i) $n(t)$ being non-activated and ii) $\left[N_{0}-n(t)\right]$ activated (by activated, we mean they present a configuration with a local shear component i.e. they form a Smd). Using Boltzmann statistics, it can be calculated [2b] that the application of a stress $\sigma$ leads to a new equilibrium corresponding to further activation of $\Delta N$ defects with :

$$
\begin{aligned}
& \Delta N=n(0)-n(\infty)=\alpha N_{0} f \sigma v_{\mathrm{a}} / k_{\mathrm{B}} T \\
& \text { and } \quad \alpha=1 / 4 \\
& \text { if } \quad \Delta u=0 \\
& \alpha=\exp \left(-\Delta u / k_{\mathrm{B}} T\right) \text { if } \Delta u>k_{\mathrm{B}} T
\end{aligned}
$$

$\Delta u$ is the energy level difference between both nonactivated and activated states.

The general equation describing the rate of activation (or rate of formation of Smd) is equation (2) i.e. :

$$
\begin{aligned}
& \mathrm{d} n(t) / \mathrm{d} t= \\
& =-[n(t)-n(\infty)] / \tau_{1}+[n(0)-n(t)] / \tau_{2}
\end{aligned}
$$

where $n(t)=n(0)$ at $t=0$ when the stress $\sigma$ is applied and $n(\infty)$ is the equilibrium population of activated state corresponding to this stress (notice that $n(0)=N_{0} / 2$ if $\left.\Delta u=0\right) . \quad \tau_{1}$ and $\tau_{2}$ being replaced by $\tau_{1}(t)$ and $\tau_{2}(t)$ respectively, equation (A.2) is not easy to integrate. As $\tau_{2}(t)$ is much higher than $\tau_{1}(t)$, we propose to consider two limit cases :

i) For shorter time $\left(\tau_{2}(t) \gg t>\tau_{1}(t)\right)$, the last term of equation (A.2) can be neglected so that this equation becomes :

$$
\mathrm{d} n(t) / \mathrm{d} t=-[n(t)-n(\infty)] / \tau_{1}(t) .
$$

Equation (A.3) which describes the anelastic behaviour, can be integrated giving :

$$
\begin{aligned}
& n(t)=n(\infty)+ \\
& +\Delta N^{*} \exp \left[-\left(t / \tau_{\max }\right)^{1-\mu_{1}} /\left(1-\mu_{1}\right)\right] .
\end{aligned}
$$

The formation of a Smd and its subsequent expansion induce a local shear $\Delta \gamma$ concerning a volume of matter $v_{1}$; this results in a macroscopic strain which is, in a macroscopic volume of matter $V_{0}$, equal to $\gamma_{1}=\Delta \gamma * v_{1} / V_{0}$.

It is worthy to note that $v_{1}$ is at least equal to the volume of the defect where the Smd is nucleated but may be larger when the Smd is expanded. By taking into account the rate of nucleation of Smd in the volume $V_{0}$, we obtain the strain rate :

$$
\dot{\gamma}_{\text {anel }}=\left(\Delta \gamma v_{1} / V_{0}\right) *[n(t)-n(\infty)] * V_{0} / \tau_{1}(t)
$$

giving with equation (A.4) :

$$
\begin{aligned}
& \dot{\gamma}_{\text {anel }}=\Delta N v_{1} \times \\
& \quad \times \exp \left[-\left(t / \tau_{\max }\right)^{1-\mu_{1}} /\left(1-\mu_{1}\right)\right] / \tau_{1}(t) .
\end{aligned}
$$


Finally, integrating equation (A.5) and using equation (A.1) give the anelastic compliance :

$\dot{J}_{\text {anel }}(t)=A\left\{1-\exp \left[-\left(t / \tau_{\max }\right)^{1-\mu_{1}} /\left(1-\mu_{1}\right)\right]\right\}$

with

$$
A=\alpha N_{0} \Delta \gamma f v_{\mathrm{a}} v_{1} / k_{\mathrm{B}} T .
$$

ii) For longer time $\left(\tau_{1}(t)<t \leqslant \tau_{2}(t)\right), n(t)$ is about $n(\infty)$ and the first term of the right hand side of equation (A.2) can be neglected so that we have an equation describing the viscoplastic behaviour :

$$
\mathrm{d} n(t) / \mathrm{d} t=[n(0)-n(\infty)] / \tau_{2}(t) .
$$

For each Smd in the volume $V_{0}$ having expanded until annihilation of the line $C_{\mathrm{n}}$, we have a macroscopic strain $\gamma_{2}=\mathcal{A}\langle\mathrm{b}\rangle / V_{0}(\mathcal{A}$ is the mean area swept by the loop $C_{\mathrm{n}}$ when it has covered the mean distance $l_{c}$ and $\langle b\rangle$ is the mean value of the shear vector).

Here again, by taking into account the rate of annihilation of lines bordering Smd in the volume $V_{0}$, we obtain the strain rate :

$$
\dot{\gamma}_{\text {viscopl. }}=\left(\mathcal{A}\langle b\rangle / V_{0}\right) * \mathrm{~d} n(t) / \mathrm{d} t * V_{0}
$$

which gives, with equation (A.7) :

$$
\dot{\gamma}_{\text {viscopl. }}=\mathcal{A}\langle b\rangle * \Delta N / \tau_{2}(t) \text {. }
$$

Let us remark that $\Delta \gamma v_{1}$ is lower than, but not too different from $\mathcal{A}\langle b\rangle$ so that we shall admit both quantities are similar. Thus, the viscoplastic compliance can be written :

$$
J_{\text {viscopl. }}(t)=\left(A /\left(1-\mu_{2}\right)\right) *\left(t / \tau_{\max }\right)^{1-\mu_{2}} .
$$

A complete expression for the total compliance is given by summing equations (A.6) and (A.8) which means that the error resulting from their application in the whole time scale, is neglected as some compensation occurs between the error in equation (A.6) (the compliance is underestimated when $0<t \ll \tau_{\text {max }}$ ) and that in equation (A.8) (the compliance is overestimated when $0<t \leqslant \tau_{\text {max }}$ ).

Finally, introducing the elastic compliance, we have :

$$
\begin{aligned}
& J(t)=1 / G_{1}+A \times \\
& \times\left\{1-\exp \left[-\left(t / \tau_{\max }\right)^{1-\mu_{1}} /\left(1-\mu_{1}\right)\right]\right\}+ \\
&+\left(A /\left(1-\mu_{2}\right)\right) *\left(t / \tau_{\max }\right)^{1-\mu_{2}}
\end{aligned}
$$

\section{References}

[1] FERRY, J. D., (a) Viscoelastic properties of polymers (J. Wiley) 1980 ; (b) ibid. p. 348 ; (c) ibid. p. 271.

[2] WARD, I. M., (a) Mechanical properties of solid polymers (J. Wiley) 1983 ; (b) ibid. p. 144.

[3] McCrum, N. G., Read, B. E. et Williams, G., Anelastic and dielectric effects in polymeric solids (J. Wiley) 1967.

[4] De Gennes, P. G., Scaling concept in Polymer physics (Cornell Univ. Press) 1979.

[5] Perez, J., Revue Phys. Appl. 21 (1986) 93.

[6] Perez, J., ICIFUAS, URBANA (USA), 1985.

[7] Аdam, G. and Gibbs, J. M., J. Chem. Phys. 43 (1965) 139.

[8] JohaRI, G., Plastic deformation of amorphous and semi-crystalline materials, Ed. B. Escaig and C. G'sell (Editions de Physique, Les Ulis, France) 1982, 109.

[9] Perez, J., Cavaille, J. Y., Etienne, S. et FouQuet, F., J. Phys. Colloq. France 41 (1980) C8-850.

[10] Perez, J., Fouquet, F. et He, Y., Phys. Status Solidi (a) 72 (1982) 289.

[11] Perez, J., Acta Metall. 32 (1984) 2163.

[12] Palmer, R. G., Stein, D. L., Abrahams, E. et Anderson, P. W., Phys. Rev. Lett. 53 (1984) 958.

[13] Ngai, K. L. and White, C. T., Phys. Rev. B 20 (1979) 2475.
[14] Decroix, J. Y., Thèse Lyon (1974).

[15] DoI, M. and Edwards, S. F., J. Chem. Soc. Faraday Trans. 274 (1978) $1789 ; 274$ (1978) $1802 ; 274$ (1978) 818.

[16] Salvia, M., Thesis Lyon (1982).

[17] Cavaille, J. Y., Jourdan, C., Kong, X. Z., Perez, J., Pichot, C., Guillot, J., Polymer 27 (1986) 693 ;

Cavaille, J. Y., Thesis (1987).

[18] Cavaille, J. Y., Jourdan, C., Perez, J., MonNERIE, L. and Johari, G. P., J. Pol. Sci. B 25 (1987) 1235.

[19] Roberts, G. E. and White, E. F. T., The physics of glassy polymers, Ed. R. N. Haward, Appl. Sci. Phys. (1973).

[20] Cavaille, J. Y., Etienne, S., Perez, J., MonNERIE, L. and JohARI, G. P., Polymer 27 (1986) 549.

[21] PlazeK, D. J., in Relaxation in complex systems, Eds K. L. Ngai and G. B. Wright (Off Naval Research, Arlington) 1984, p. 83.

[22] Owen, A. J. and Bonart, R., Polymer 26 (1985) 1034.

[23] Rammal, R., J. Phys. France 46 (1985) 1837.

[24] NGAI, K. L. and Wright, G. B., Ed., Relaxation in complex systems (Off Naval Research, Arlington) 1984. 